\title{
ANTIMICROBIAL RESISTANCE AND ANTIMICROBIAL STEWARDSHIP PROGRAMS: BENEFITING THE PATIENT OR THE POPULATION?
}

\author{
Alberto Giubilini \\ Oxford Martin School \\ University of Oxford
}

Antimicrobial resistance kills people. According to a recent estimate, "700,000 people die of resistant infections every year", and "by 205010 million lives a year are at risk due to drug resistant infections, as are 100 trillion USD of economic output"1. Today, "bacteria are resistant to nearly all antibiotics that were earlier active against them"2. For all these reasons, antimicrobial resistance is considered a "slowly emerging disaster"3 and a "global health security issue" ${ }^{4}$. The prospect we are facing is that of a "post antibiotic era" ${ }^{5}$, in which we risk losing the availability of effective antibiotics and, with them, of medical interventions that require effective antibiotics, such as chemotherapy, transplantation, and major surgery.

One of the causes of antimicrobial resistance is antimicrobial consumption. As put by the Center for Disease Control and Prevention, "the use of antibiotics is the single most important factor leading to antibiotic resistance around the world: simply using antibiotics creates resistance"6. According to the 2016 Review on Antimicrobial Resistance commissioned by the UK Government, "more consumption of antibiotics directly leads to more resistance" . This is because microbes that are not killed by antimicrobials are more likely to reproduce and proliferate and to pass their resistance genes on to other microbes. Thus, exactly like the consumption of many other finite resources, antibiotic and antimicrobial consumption gives rise to the collective action problem known as "tragedy of the commons": consuming a certain resource, such as antimicrobials, is in an individual's best short-term interest, but many instances of individual consumption erode the resource and therefore conflict with the collective interest in preserving the good. How to solve a tragedy of the commons? As is the case with the consumption of other finite resources, the answer is quite simple: by rationing the resource.

Simon Oczkowsi's article in this issue of the journal addresses the problem of whether rationing antimicrobials through stewardship programmes in U.S. hospitals, or antimicrobial "bedside rationing”, is morally permissible. Oczkowski starts off by arguing that antimicrobial stewardship programmes (ASPs) recommended by the Infectious Diseases Society of America (IDSA) which aim at narrowing the spectrum, reducing the dose, and shortening the duration of antimicrobials used in clinical practice in order to contrast the development of antibiotic-resistant organisms (AROs) do constitute a form of bedside rationing: ASPs require clinicians to reduce individual patients' access to a treatment that is in the patients' best interest in order to promote the interests of others, i.e. those who in the long term would benefit from the availability of effective antimicrobials. Oczkowski's main thesis is that this form of bedside rationing of antimicrobials is "fair and ethically sound" (p. 1) even if it doesn't promote the short-term best medical interest of the individual patients who are prevented from using antimicrobials. This observation is important because the fact that rationing a service conflicts with an individual's best medical interest is often seen by clinicians as a negative aspect of rationing, which gives rise to concerns about ASPs. Oczkowsi' article aims at salvaging the concept of rationing by arguing that the 
"bedside rationing" of antimicrobials is consistent with Norman Daniels' 'accountability for reasonableness" model, and therefore it is ethical and fair. According to Daniels' model, a rationing process is considered fair if it meets four requirements. These are 1) a publicity condition, according to which decisions and rationales about rationing antimicrobials must be publicly accessible; 2) a relevance condition, according to which reasons and principles that can be agreed on are relevant; 3) an appeals/revision condition, according to which there must be a mechanism in place for resolving disputes or revise a decision; and 4) an enforcement condition, according to which there must be a regulation in place to ensure that the preceding conditions are met. Oczkowski argues, convincingly, that IDSA's guidelines for ASPs fulfil all of these conditions.

One aspect of ASPs that Oczkowski emphasizes is that the type of rationing recommended by ASPs is a "bedside" type of rationing, which means that according to ASPs it is clinicians who have control over, and the responsibility for, the modality of antimicrobial administration or non-administration to their patients. In other words, ASPs make clinicians responsible for the decisions of whether and how (not) to prescribe antimicrobials. As put by Oczkowski, "the treating physicians jointly have the discretion to prescribe or limit the therapies used, and decide in what cases rationing is to be used" (p.2). Oczkowski argues that "the 'publicity condition' in ASPs refers to the HCPs involved in the circle of care of a patient, rather than the patient himself or herself" (p. 2). According to Oczkowski, clinicians should make decisions from behind a Rawlsian veil of ignorance, i.e. "as if they were unaware whether they personally would have to live with the consequences of that decision" (p.2). Ockowski seems optimistic about the prospect that this method will lead to "generally agreed-on principles and values, including the prevention of the development of AROs, reduced healthcare costs and maximising of clinical benefit for individual patients” (p.2)

However, one might wonder whether it is justified to burden clinicians, and exclusively clinicians, with the responsibility of making rational and ethical decisions aimed at balancing individual and collective interests. Besides, without some more precise guidance as to how exactly each a clinician is supposed to ration antimicrobials, one might ask whether the path to "generally agreed-on principle" will be as straight and smooth as Oczkowski seems to assume. The two problems - about the responsibility of clinicians and about the agreement on principles - are closely related. One might legitimately claim that we would be putting too much pressure on clinicians when it comes to choosing between their patients' best medical interest and societal interest in preserving antimicrobial effectiveness, and that clinicians might not be able to get to generally agreed-on principles that balance the three desiderata of 1) prevention of AROs, 2) reduced healthcare costs, and 3) maximization of clinical benefit for individual patients. It seems reasonable to suppose that clinicians would favour the last desideratum, and that they might do it by remaining within the accepted ethical principles of their profession. After all, a prima facie principle of good clinical practice is that clinicians should act in view of promoting their individual patients' best medical interest. It seems plausible to suppose that clinicians would be reluctant to make decisions from behind a veil of ignorance and restrict antimicrobial prescription. In the absence of guidelines and policies that put explicit and precise limits on clinicians' freedom to prescribe antimicrobials, the ideal method of making decisions from behind a veil of ignorance is likely to create serious ethical 
dilemmas that clinicians might struggle to solve. Thus, there is perhaps more than simply issuing ASPs that institutions can do in order to encourage individual clinicians to ration antimicrobials. For example, one might wonder whether ASPs should include an upper limit to the amount of antimicrobials that each clinician can prescribe over a certain period of time; in this way, whether they want it or not, clinicians would not be allowed to prescribe more than their allotted amount of antimicrobials. Another solution could be to tax antimicrobials, so as to achieve two goals: first, patients who are prescribed antimicrobials would contribute to internalizing the negative externality produced by antimicrobial consumption, for example if revenue from the tax were destined to fund research and development of new antimicrobials; second, patients' expectations to be prescribed antimicrobials for any type of infections would be reduced, and with them also the pressure on doctors to overprescribe antimicrobials would be diminished.

Another aspect of ASPs that Oczkowski emphasizes in conclusion of the article is the fact that "ASPs withhold or withdraw care that is in the individual patient's best interest and puts them at short-term risk, although there is a theoretical long-term benefit if those risks are well managed and the patient survives” (p. 3). The short-term risk to individual patients means that ASPs can be considered a form of rationing, in spite of the long-term benefit to the population. However, the patient, although she is prevented from accessing a medical treatment she needs, would benefit from ASPs to the extent that she is a member of the population that benefits from the long-term preservation of antimicrobial effectiveness. In particular, as Oczkowsi notes, "ASPs have resulted in improvements in patient outcomes, such as a lower risk of Clostridium difficile infections secondary to inappropriate antibiotic use” (p. 3). This consideration about the long-term benefit to patients, although it does not alter the fact that withdrawing or withholding of antimicrobials is a form of rationing (i.e. something that runs counter patient's short-term best interest), strengthens the justification for the implementation of rationing through ASPs. This is because the individual patient is not only making a sacrifice in order to benefit other people which might be seen as supererogatory -, but she makes a sacrifice that in the longrun will contribute to benefiting herself. Thus, the long-term benefit to the patient can be invoked as a further reason that strengthens the ethical justification for the implementation of ASPs. We can therefore agree with Oczkowski conclusion that ASPs are "an exemplary demonstration of how bedside rationing can be procedurally fair and lead to excellent clinical outcomes” (p.3).

\section{REFERENCES:}

1. O’Neill, J. Tackling Drug Resistant Infections Globally: Final Report and Recommendations. The Review on Antimicrobial Resistance 2016, available at http://amrreview.org/sites/default/files/160518_Final\%20paper_with\%20cover.pdf, last access 10 Sept 2017

2. Herrmann, M, Laxminarayan R. Antibiotic effectiveness: new challenges in natural resource management, Annu Rev Resoue Econ 2010; 2: 1-14 
3. Viens, AM, Littmann J. Is antimicrobial resistance a slowly emerging disaster? Public Health Eth-UK 2015;8: 255-265

4. Balasegaram, $M$ et al. The global innovation model for antibiotics needs reinvention. J Law Med Ethics 2015;43, S3: 22-26

5. Alanis, AJ. Resistance to antibiotics: are we in the post-antibiotics era? Arch Med Res 2005; 36: 597-705

6. CDC (Center for Disease Control and Prevention). About antimicrobial resistance, updated 6 Apr 2017, available at https://www.cdc.gov/drugresistance/about.html, last access 10 Sept 2017 